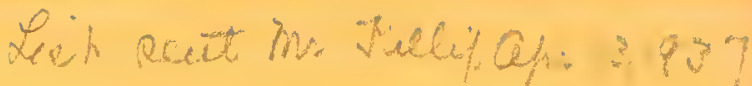

Venturi Plants (Mar. 18, 1937).

285 Tropaeolum caplliare Buch. (Iabelled T. argentinum)

705311

,

II

11

n

"1

8805

$\|$

It

II

II

$\|$

517 J Jerusia sheranitice (St.-H1I.) Juss.

1518 Polygala I1noides Polr.

2028 " chloroneura Grín.

4671 " 1 "

3159 "

7830 Gh1ropetalun tricuerlantum (Lan.) Juss.

8186 Jetropha urens J.

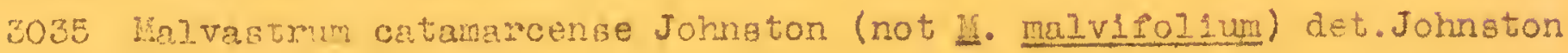
7531 Hyparlom connatun Len:

8060 Justiclo pruc1f?ora Gris.

w925 "l nenoreIs LiIlo 


\section{Venturi Plants}

55ar Phyllostylon rhamoldes (Po1s.) Taub.

Lablatae det. Dping

1340 Salvia GiIIIesli Benth.

4880

w

$n$

4956 " stachyojfolia Benth.

5544 Hyptis Iapacea Benth.

5947 Mentha viridis L.

5958 Myptis nutablils (RIch.) Brig.

7594 Scuteliaria breviflora Behth.

fil? Leoechnina pravenlens (Rerel) spl.

317. Fotis mutabils PRich.) I3rio.

092 Gel wa g tachy lifolat Benth.

I7z Hoptis leppece Benth.

2007 Lepachinia florthwia (Benth.) Eol. 
EUPATORIEAE IN A COLLECTION RHCHIVED FROM MR. S. VEVTURI APR. 4, 1929. AS DETERINAED BY B. I. ROBINSON

5802 Eupatorıum inulaefolium HBK. f. suaveolens (HBK.) Hieron.

5855 "
6081 " $\quad$ Arnottii Bak.
6140 Ageratum conyzoides L.
$617 \%$ Eupatorium laevigatum Lam.
6181 " "
$7070 \quad$ Lquarroso-ramosum Hieron.


"ro. venturi.

Received at Gray Terharium Feb. 1?, 1930.

Proptiel $\left\{\begin{array}{r}362 \\ 7278 \\ 5679\end{array}\right.$

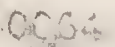

$9 \cos ^{2}: 3$

9030

3477

2uीn

rtovia broviaristi tia r.

Jopet, rium tueumanense Iilzo Robinson

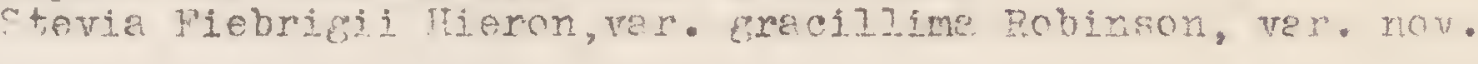

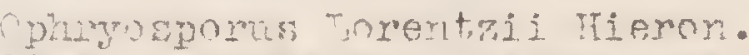

- tevia yeconerms itieron, var. suberlendulose Hieron.

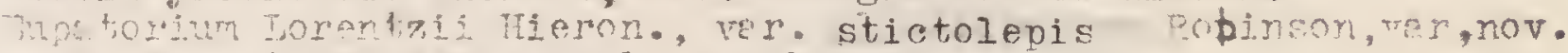
tracumenense Ijizlo \& Pobinson

5.189 Pournefortia Ti27oj tometon

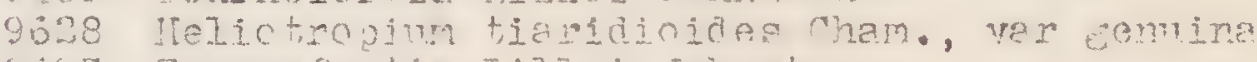

6.7 Tourefontia Ijisci Tohnston

3648 " "a $7 \%$ manni De.

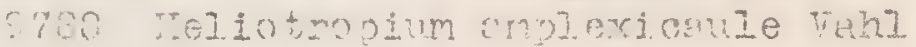

9197 splentum Cacusseniz Iternn. probeby

9203 Polrpocium Tweedienum Hook.

0517 Splenium sulcatixm Inam.

9578 diantopsis regularis (Yze.) Koore

9579 isnleninm sulcetum Lam.

9.85

9587

9588

9590

9626

3639

$97: 6$

serra Iangad. \& Fisch.

mopteris pecete (L. r.) Féo. var.

Isplerilum praemorsum ?w.

sul.eatum Iam.

" Clamsseni Hieron. probably

Mtopteris Eregilis (J.) Bernh.

polypodium lycopodioldes $\mathrm{I}$.

Ctevia vaga Griseb.

4868

$"$ breviaristata $H . \& A$.

- mercedensis Hieron., tar. Elenditecte Pobinson 


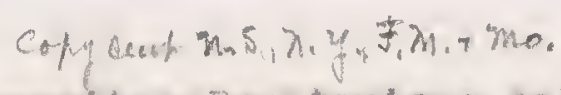

$7 \min .7 .195 \%$

Compositac-Hupator1eas col1. by S. Ventur1 det. by B. L. Roblman

101 upatorlun hecatanthum (DC.) Dak.

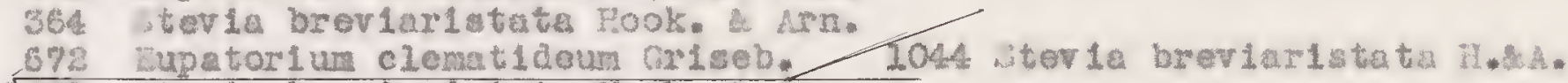

1051 stevla brevlarlistuta llook. bron.

1205 Mikmia saltens 1 s Meron.

1538 itevis extuad Iteron.

1815 Eupator1 um srotiti Bek.

1834 itev1e brovlaristata Wook. G Arn.

1853 hupatoni um rnottianu Griseb

Eg66 ttev1a brev1aristata look. Ar. Mr.

3036 " I11101 Robinson

3079 " vaca criseb.

3152 " breviariateta look. L Mrn.

gio5 jupatoriun Lorenta11 ideron.

3563 , tevia vaga Criscb.

3304 Lupatorius sol1arafnoldes mor.

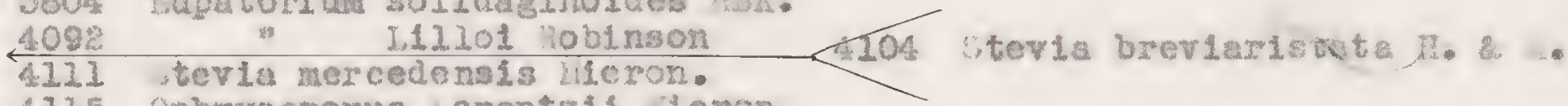

4115 ophryosporus Lorontz11 ileron.

412 stenia efruan vieron.

4161

4736

4730

4868

4920

5003

5007

5031

5496

5531

5802

5875

5018

5833

5953

6008

6072

6081

$612 \mathrm{c}$

6168

7353

7996

8054

8635

"Jaconensia Micron. var. Eubeglandulase Mieron.

* Chanedrys criseb.

" Vaca criseb.

" mercedens 1s Lieron. var. Eland1tacta Robinson

Dupatorium Arnottianum oriseb.

Stevia breviaristatu Hook. \& Rn.

hupatoriun patens D. Hon ver. tomentosura licron.

ophryosporus charus (Griseb.) slcxon.

Ageratum conyzoldes I.

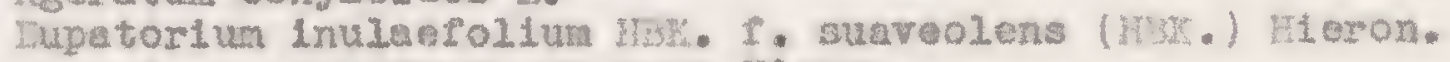

h. tev 1 a vaca Griseb.

" brev1ar13tata liook. A Arn.

Iupator lum Arnottianua Griseb.

stevla effusa Mleron.

" yaconens ls theron. Var" subesiandulosa Mleron.

Dupator 1 u Arnott11 Mek.

tevia breviarietuta Hook. * Arn.

- Mercelensis Hieron.

Lupator lum Arnottianum Griaeb.

wevila eifusa Bieron.

* yaconchsis Heron. var. aristrera hobinson

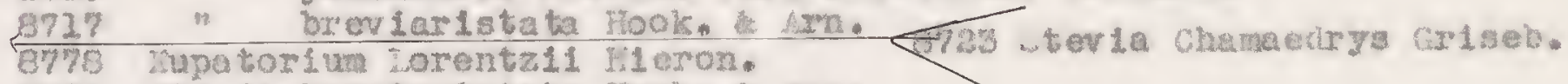

8806

8870

$927 \%$

9386

10,013

10,219

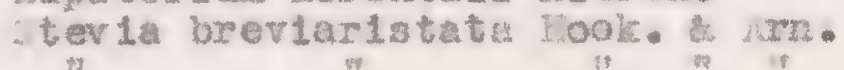

- upa torlum azangaroense 1 ch.-31p.

" Iorenta11 ileron. var. st1ctoleg1d lobinson

"tev 1 a chamalarys Griseb.

10,220

" 
5453 hechmea polys tachya (Vell.) Lez (as Ae. Brandiceps)

1515 T11landsia cap1llaris R.\&W. forma incana (G111.) L.B. Smith

957

1917

1903

54.76

1900

2880 pulchella llook. var. surinamonsis (M1q.) $\mathrm{Vez}$ capillaris R.e. . Forma incana (Gill.) L.5.Smith *

ค

(⿻) 11

$n$

i"

*⿻1 cordobensis (Hieron.) L. . Smith

Dyckia floribunda Griseb. 


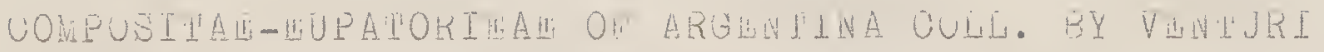

$$
\text { (Received in sumer of 1930) }
$$

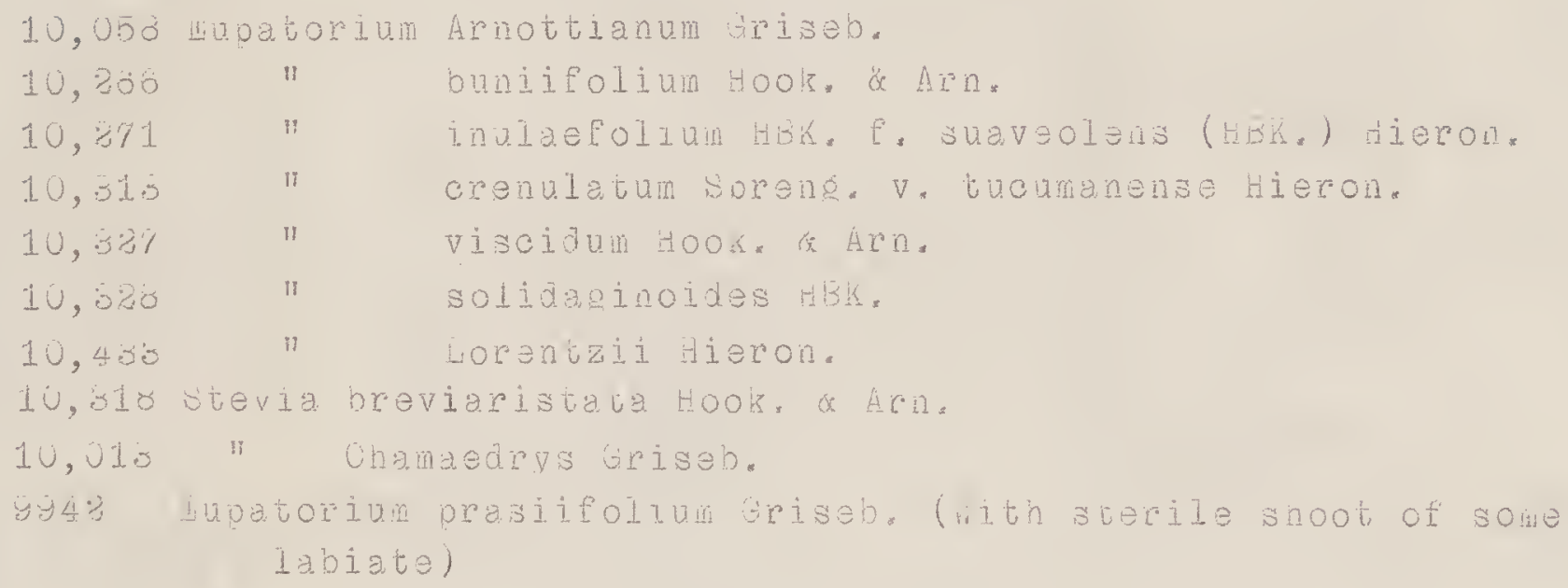




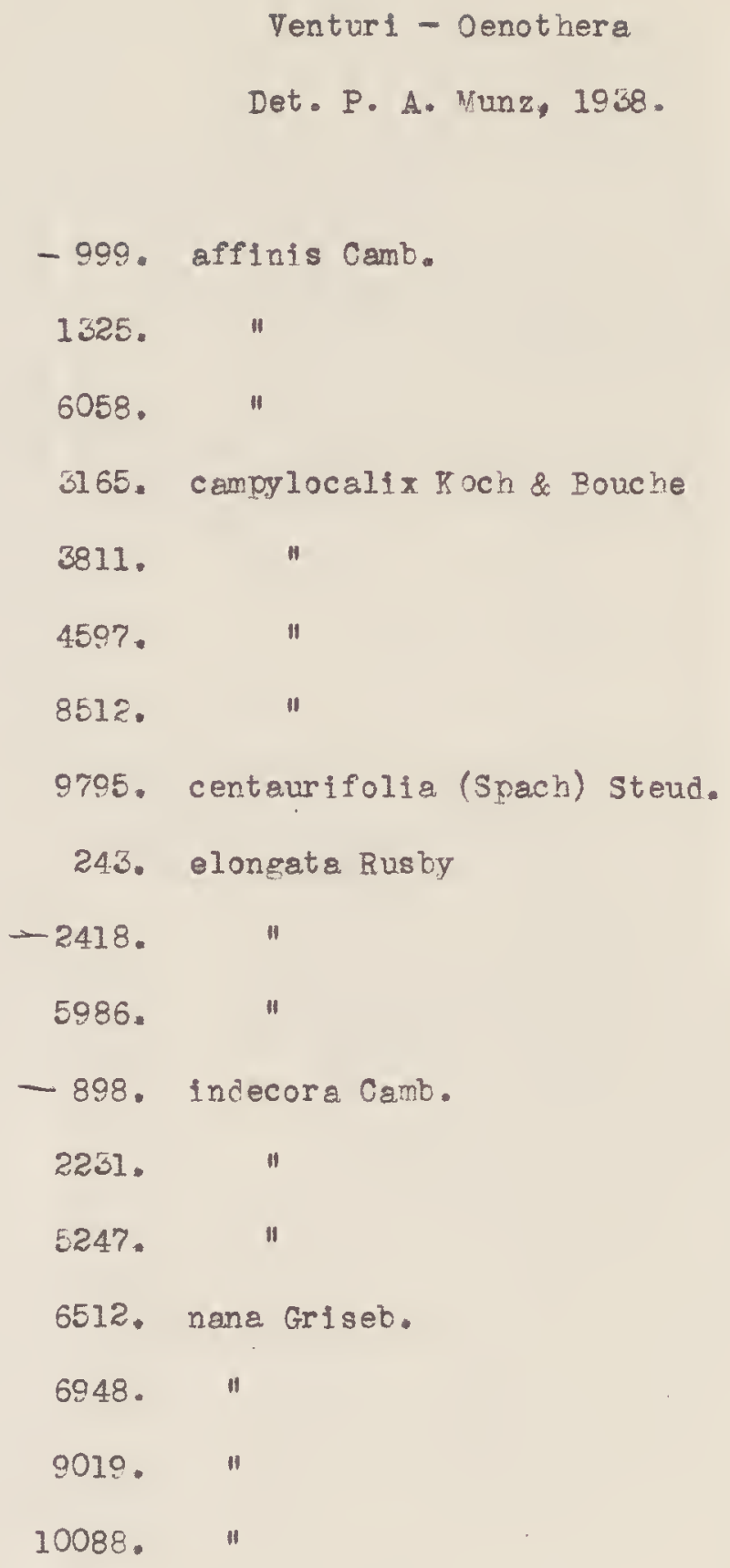




\section{Venturi - Plantago}

Det. Pilger, 1935.

Reported by U. S. National Museum, October 1935.

251. P. hirtella Kunth (broader leaves! and P. tomentosa Lam. 522. P. rugosa Lam.

2184. P. "I. "

3085. P. lorentzii Pilg. (not sericea)

3097. P. venturii Pilg. n. sp.

3174. P. lorentzii Pilg.

3561. P. tomentosa petiolata Pilg.

3914. P. " Lam. var.

3915. P. hirtella f. minor Pilg.

4393. P. candollei Raf.

4565. P. monticola crispula Pilg.

4716. P. hirtella Kunth

4721. P. lorentzii Pilg., ad P. monticola vergens

6946. P. monticola sericans Pilg.

6947. P.

7058. P. venturii Pilg. type

7394. P. niederlienii Pilg.

8508. P. orbignyana Steinb.

8510. P. monticola Dene

8511. P.

8748. P.

II

9008. P. niederleinii Pilg.

9586. P. hirtella Kunth

10109. P. monticola sericans Pilg.

10110. P. orgignyana Steinb.

10276. P. monticola sericans Pilg. 
Venturi

Reported by U.S.N.M. Jan. 1939

Caryophyllaceae (Det. Mattfeld)

414. Cerastium rivulare Camb.

904. " "

2200. Stellaria media (I.) Vill.

2634. Arenaria lanuginosa (Mx.) Rohrb.

2900. Drymaria ovata Willd.

2983. Cerastium rivulare Camb.

3078. Cerastium arvense $I$.

3110. Arenaria palustris Naud.

3459. Stellaria media Vill.

3460. Cerastium rivulare Camb.

3912. "

4248. " "

4421. Drymaria ovata Willd.

4578. Cerastium arvense $I$.

4612. " "

4617. Arenaria achatlensis Griseb.

4652. Arenaria megalantha var. tucumanensis (Griseb.) Williams

4761. Cerastium vulgatum var. peruvianum Williams

5104. Drymaria cordata (I.) Willd.

5878. Cerastium rivulare Camb.

6067.4 "

6370. Pycnophyllum convexum Griseb.

6371.

6441. Arenaria lanuginosa (Mx.) Rohrb.

6442. " palustris Naud.

6614. Cerastium vulgatum var. peruvianum Williams

8543. Arenaria rivularis Phil.

8611. Pycnophyllum molle Remy

8682. " " $8853 . \quad$ "

9482. Arenaria megalantha var. tucumanensis (Griseb.) Willians

9554. Sagina decumbens T. \& G.

10027. Cerastium rivulare Camb.

10076. Stellaria cryptopetala Griseb.

10081. Cerastium vilgatum vulgatum var, peruvianum Williams

10082. Stellaria aphanantha Griseb.

10083. Pycnophyllum mucronulatum Mattf.

Frankeniaceae (Det. Mattfeld)

10078. Anthobryum triandrum (Reny) Surgis 
Venturi (cont.)

M1 cellaneous faril1es

992. Humex obovatus Danser (Det. Rechinger)

1008. Seneclo canablnaefollus llook. Arn. (Det. Greenman)

1753. Goventa tingens F. \& T. (Det. W1111ara)

2288. Rumex conglomeratus Miers. (except large leaves; det. Rechinger)

2572. Senecio bowmani R. F. Iries (Det. Oreenman)

4952. Hypseochar1 arydalifolla I. Knuth (Det. Knuth)

5014. Rumex crispus L. (Det. Rechinger)

5136. Stylosanthes gulanens1s (Mub1.) Sw.

5375. Rumex obovatus Danser (Det. Rechinger)

5378. Melloa populifolla (DC.) Bur. (Det. Selbert)

6720. Diontea scoparla (0111. \& Hook.) Mlers (Det. Moldenke;

alst. as Verbena scoparia)

7561. Amphilophium pan1culatum (L.) H.H.K. (Det. Selbert)

8609. Fypseocharls arydalifolla R. Knuth (Det. Knuth)

9471. Senecio bowman1 H. M. Fries (Det. Oreenman)

9662. Melloa populifolia (DC.) Iur. (Det. Seibert)

10008. Senecio canabinaefoliu H. A. (Det. Greenman) 
Ventur1 (Eeported by U.S.I.M. June 1938)

Det. Patel Aellem

447. Chenopodium arralo i.

3268. ambrosiolaes subsp. burkertil Aellen

1388. parnolosum hoq.

435. sabrosiol det I.

564. Suedia

Det. I. U. Johnston

1912. Aneenilis pumila $\mathrm{SW}$.

2038. Spirasa truabergi1 $\$ 14 \mathrm{eb}$.

3125. Glie grec111 (Dougl.) trook.

3373. Crasmula near closinna Gay

4046 . Anemone cicutifolla Johnst.

4052. Chaet anther a

4664. Narerr icarpus palue I jugat un Johnst.

4763. Sext frafa peruviana sternb.

5216. Torminalis triflora Grisob.

6350.

"

5472. Jatropha cf. pachypoda Pax

5643. Petunie perviflora Juss.

5E47. Phyllostylon rhamnoldes (Polr.) Taub.

5585. Coccoloba cordata Cham.

5595. Conolobus australis Malme

6360. Nareyr1carpus inermis Johnst.

6438. Mixture of Mareyrlcarpus tucumanus Johnst. and Acesmia sp.

6633. Mareyricar pus inermis Johnst. (type)

6682. Aresal a schickendanz11 arisob.

7534. Kenodora heseler lana chod.

7564. ct. Xylosme pubescene Griseb.

7588. Bumella Cf. excelsa DC.

7948. Talinum polygaloides G1111 es

8134. Nareyr 1carpus tucumenus Johnst.

8540. "Inermis Johnst.

8624. " cristatus Brition

8787. Hy per 1cum brevistylum Chois.

9190. Henodora haselerlena Chod.

9792. Coccoloba cordata Cham.

10140. Port vleca 
Det. R. Tnuth; reported by U.B.H.M. Mar. 10, 1937

\section{Oxal1s}

2101. corniculata L.

2813. pubescens H.B.K.

- 2895. tuberosa Mol.

3155. chlch 1gestens 18 Inuth (typo)

-4031 . Eyrorhiza Bert.

4068. tuberosa $\mathrm{Nol}$.

4425. pubescens स.В.K。

44258. membranifolia Rnuth (type)

462\%. elegantula Tnuth

5896. pubescens H.B.K.

- 6066. refracta St. Hil.

7440. papll Ionacea Hoffmans.

8090. macachin Arech.

\section{Geranfum}

-2218. carolianum I.

- 2377. venturianum Tnuth (typo)

$-2667$.

-2984. patagonicum Hook. $f$.

3034 . "

3109.

$-3269$.

- 3089. mexicanum minoriflorum (Briq.) Xnuth

- 3645. delphinifollun Knuth (type)

-3919. dessectrm I.

- 4026. patagonicun Ilook. $f$.

$-4036$.

- 4619. "

-4650 .

- 4807. sessilifiorum Car.

- 5943. venturianum Knuth 6902. sess1liflor um Cav.

- 7723. venturienum Tnuth

7842. ubsericeum Inuth

8626. sessiliflorum Cav.

8696.

9223.

9355.

"

*

-9848. venturianum Fnuth 


\author{
Venturi \\ Cyperacoae, det. R. Gross \\ (Reported by U.S.N.M. June 1939)
}

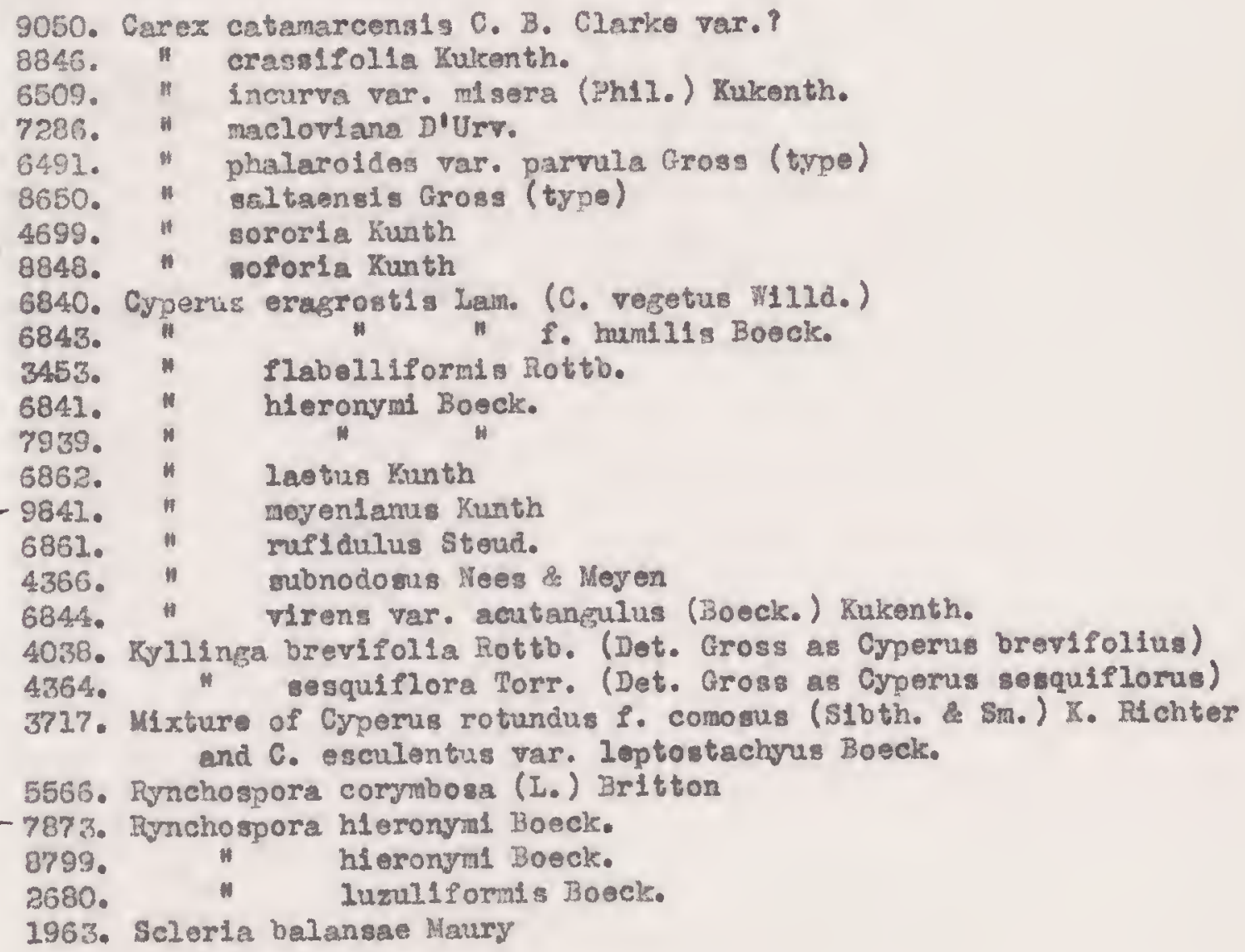


Venturi, Lobeliaceae, det. Wimmer

2992. S1phocampylus cuspidatus tucumanus Wimmer (type) 4063. ต . *

5173. Lobelia cliffortiana xalapensis (H.B.K.) A. Gray 7428. Hypsela renifotmis (H.B.K.) Presl (Det. Killip) 8589 . 9040. 2

9652.

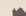

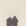

(Det. Killip)

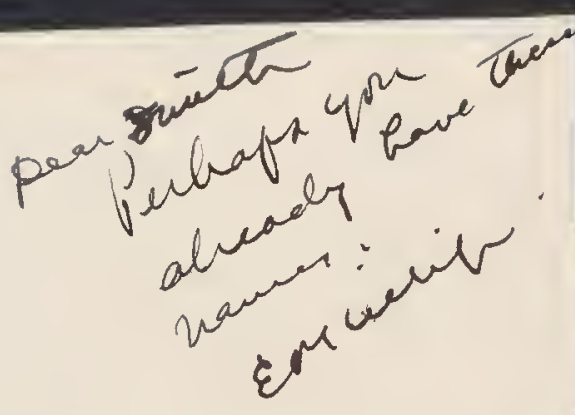




\section{Ventur1 Veleriena}

\section{Det. R.P.K1II1p}

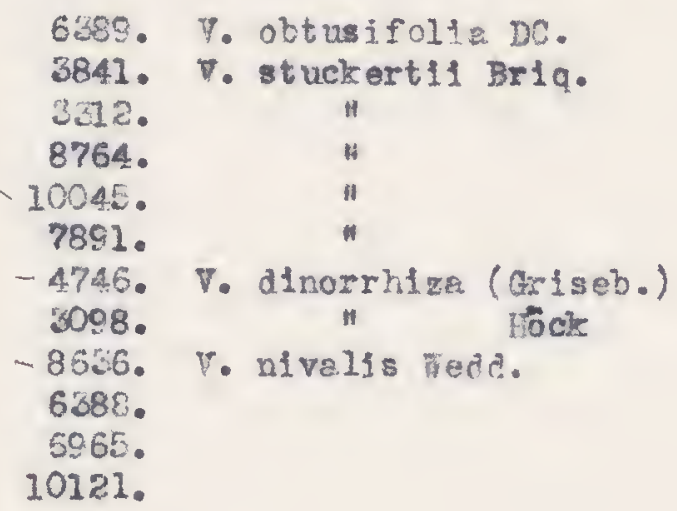


Tentur1 -

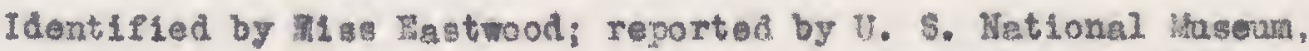
Septerabar 2, 1937

3872. F. rostrata Schlecht.

3873. 3. Sideroxylon Cunn.

3874. R. teret1cornis Srath

3875. 2.

"

n

3876. I.

3377.

n

3878. 莎.

औ

*

3888. T. tereticornis Snith

3689. x,

t?

u

3890. 3.

*

U

3891. 2. gonlocalyx F. \& M.

4993. Ieucoxylon $\bar{F}$. It.

9058. 5. robusta 5 .

9062. x. rottrate schlecht. 